EPJ manuscript No.

(will be inserted by the editor)

\title{
Strangeness contributions to nucleon form factors
}

\author{
Ross D. Young \\ Jefferson Lab, 12000 Jefferson Ave., Newport News, VA 23606 USA \\ Received: date / Revised version: date
}

\begin{abstract}
We review a recent theoretical determination of the strange quark content of the electromagnetic form factors of the nucleon. These are compared with a global analysis of current experimental measurements in parity-violating electron scattering.
\end{abstract}

PACS. PACS-key discribing text of that key - PACS-key discribing text of that key

\section{Introduction}

The determination of the strange quark content of the nucleon offers a unique probe to measure the nonperturbative structure of the nucleon. As the nucleon carries zero net strangeness, the influence of strange quarks arises entirely through interaction with the vacuum. Technically speaking, strange quarks directly probe the role of the fermion determinant in QCD. While strangeness measurements in nucleon structure have been difficult to isolate, the contribution of the neutral weak current in elastic scattering offers perhaps the most direct measurement of the strange quark content of the nucleon [1].

Here we review recent progress in the study of the strange quark contributions to the nucleon form factors. In Section 2 we discuss the theoretical developments in the chiral extrapolation of lattice simulation results that have enabled a precise determination of the strangeness form factors. An outline of this determination is provided in Section 3. In Section 4 this theoretical prediction is compared with a global analysis of the experimental measurements searching for strangeness in the nucleon.

\section{Chiral applications in lattice QCD}

The computational expense of incorporating the effects of the fermion determinant has restricted modern lattice QCD simulations to the use pion masses that are typically $m_{\pi} \gtrsim 500 \mathrm{MeV}$. Recent progress has seen nucleon 3-point functions simulated with pion masses pushing down to the $350 \mathrm{MeV}$ range [2-4], yet a reliable extrapolation in the pion mass is still required to compare with reality until the physical point is readily accessible.

Ultimately, chiral perturbation theory offers the potential to deliver model-independent quark-mass extrapolations of lattice results. As disappointing as it may be, there is mounting evidence that applications of low-order chiral expansions should be taken with serious caution beyond pion masses of the order $300 \mathrm{MeV}[5,6]$. Further, the situation could be significantly worse for observables which are particularly singular near the chiral limit, such as magnetic moments [7], charge radii or polarisabilities.

In the future, chiral extrapolations will be constrained model-independently by precision, large-volume lattice calculations in the chiral regime. Until then, one requires methods which can reliably extrapolate from the moderately-heavy quark mass regime, while maintaining all the constraints of the effective field theory. The best available solution is to reformulate the effective field theory using finite-range regularisation (FRR) [5].

In extrapolating lattice simulation results from beyond the chiral regime, one cannot guarantee that results will be independent of regularisation scheme. By choosing a particular scheme, one has necessarily introduced a model - whether it be FRR or a more traditional regularisation. The advantages of FRR have been quantitatively demonstrated for the nucleon mass. Using lattice results over the range $0.25 \gtrsim m_{\pi}^{2} \gtrsim 1.0 \mathrm{GeV}^{2}$, the FRR extrapolated nucleon mass at the physical point displays less than $1 \%$ variation associated with the truncation between successive orders in the chiral expansion. Further, the sensitivity to the choice of functional form of FRR is also less than $1 \%$ [8]. Until sufficient lattice results are available in the chiral regime, when the choice of regularisation becomes superfluous, FRR offers independent-of-model chiral extrapolations.

Because of the cost of simulating the fermion determinant, historically it has been common in lattice QCD to ignore this contribution to the path integral. This is the quenched "approximation", where the influence of quarkantiquark pair-creation in the vacuum is neglected. Fortunately, the study of the chiral extrapolation of baryon masses in quenched and dynamical simulations has revealed a remarkable phenomenological relation between these simulations. The differences between quenched and dynamical baryon masses are well described by the differences in the Goldstone boson loop corrections of the low- 
energy effective field, when evaluated with an appropriate finite-range regulator [9]. Although this is not a fieldtheoretic connection, the numerical success does mean that one has substantial confidence in obtaining physical estimates from quenched lattice results.

Beyond the baryon masses, the technique of chiral unquenching has been extended to the nucleon magnetic moments [7]. Here it was predicted that there should be very little difference in the quenched and dynamical nucleon magnetic moments over a large range of quark masses, with significant differences only anticipated near the chiral limit. These findings have been recently supported by first calculations with 2-flavour dynamical lattices [3,4].

With the success of chiral extrapolations and the estimation the effects of the quark determinant, we look to the extraction at the strangeness contributions to the nucleon electromagnetic form factors.

\section{Strangeness calculation}

Direct lattice QCD calculations of the strangeness content have been unable to produce a conclusive determination [10]. It is hoped that the next generation of calculations could shed light on this elusive signal. This may require further development of emerging lattice techniques. One potential gain could be seen by utilising background field methods [11], where a weak signal could be enhanced by coupling a strong electromagnetic field to the vacuum strange quarks. The method to evaluate the all-to-all propagator developed by the Dublin group offers significantly improved precision over traditional stochastic estimators [12], and it would be interesting to see this applied to a strangeness form factor calculation.

While awaiting the development of these techniques, one must rely on more indirect methods for an accurate extraction of the strangeness form factors. By manipulating the QCD path integral, one can isolate the various quark contributions to baryon 3-point functions [13]. Using this decomposition, combined with charge symmetry [14] and the experimentally measured hyperon radii, the sensitivity to lattice systematics can be significantly reduced [15]. The strangeness magnetic moment can be written as

$$
\begin{aligned}
G_{M}^{s} & =\frac{{ }^{l} R_{d}^{s}}{1-{ }^{l} R_{d}^{s}}\left[2 p+n-\frac{u^{p}}{u^{\Sigma}}\left(\Sigma^{+}-\Sigma^{-}\right)\right], \\
G_{M}^{s} & =\frac{{ }^{l} R_{d}^{s}}{1-{ }^{l} R_{d}^{s}}\left[p+2 n-\frac{u^{n}}{u^{\Xi}}\left(\Xi^{0}-\Xi^{-}\right)\right],
\end{aligned}
$$

where $p, n, \Sigma^{ \pm}$and $\Xi^{0 /-}$ denote the experimentally measured magnetic moments of the respective baryon. The formulae each rely on two inputs from lattice simulations. The first is the ratio $u^{p} / u^{\Sigma}$, which measures the relative strength of the valence (Fig. 1a) $u$-quark contribution in the proton relative to the $\Sigma^{+}$- or similarly $u^{n} / u^{\Xi}$ in Eq. (2). The second is ${ }^{l} R_{d}^{s}$, which describes the ratio of the strange-to-light disconnected (Fig. 1b) contributions.

Equating equations (1) and (2) and using the experimental magnetic moments produces a linear relationship

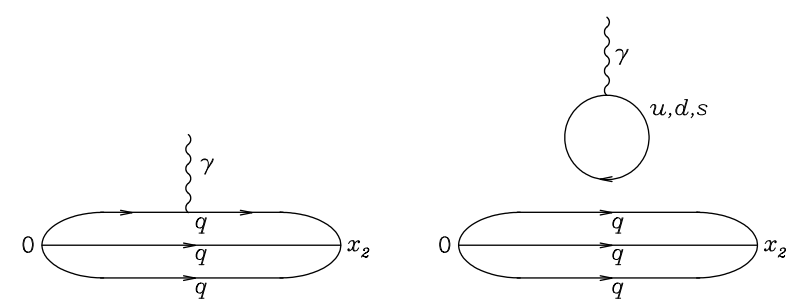

Fig. 1. Connected (left) and disconnected (right) contributions to baryon 3-point functions.

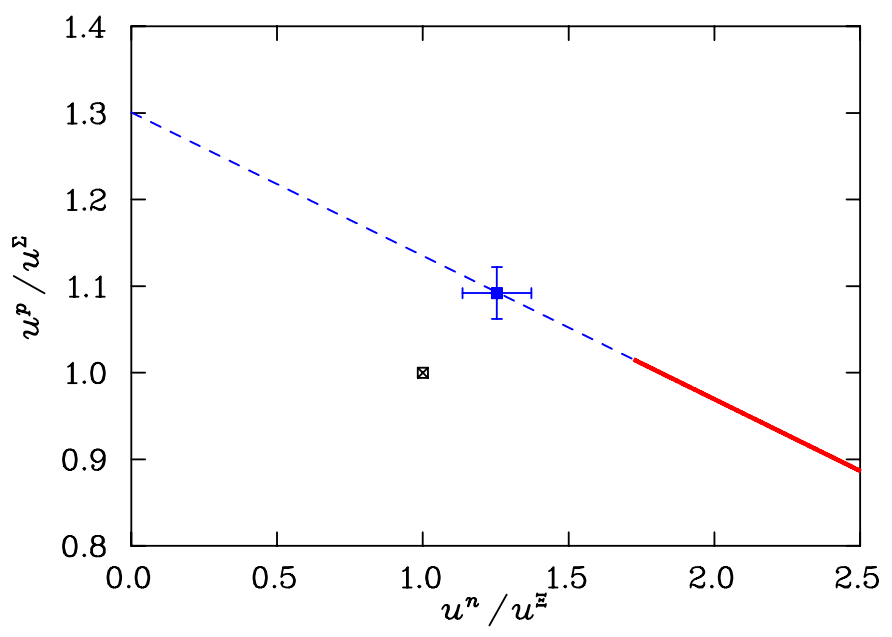

Fig. 2. The line (dashed $G_{M}^{s}(0)<0$, solid $G_{M}^{s}(0)>0$ ) indicates the charge symmetry constraint on the ratios $u^{p} / u^{\Sigma}$ and $u^{n} / u^{\Xi}$. The crossed square indicates the point corresponding to environment independent quark moments. Our determination is illustrated by the filled square [16].

between the two unknown valence ratios. This constraint, a result of charge symmetry alone, is displayed in Figure 2. The line is divided by two segments, where the sign of $G_{M}^{s}$ can be determined under the quite general assumption that $0>{ }^{l} R_{d}^{s}>1$. Recently it has been suggested that there could be a sign change in this ratio between the heavy-quark limit and naive expectations in the Goldstone boson sector [17]. Given that the properties of the kaon are much more Goldstone-like than a heavy-light meson, and that the heavy-quark limit of $\mu_{p} / \mu_{n}$ is approached very slowly [18], it should be not be expected that the strange quark could be reliably described by heavy-quark effective theory.

The techniques discussed in Section 2 were applied to determine the ratios $u^{p} / u^{\Sigma}$ and $u^{n} / u^{\Xi}$, appearing in Eqs. (1) and (2). The analysis has utilised a high-precision numerical study of the baryon electromagnetic form factors in quenched lattice QCD [19]. Upon performing finitevolume corrections, adjustments for the quenched approximation and a controlled chiral extrapolation, the resulting ratios are compared with the experimental constraint in Figure 2 [16]. The excellent agreement with the constraint from experiment is a first check on the consistency of our calculation. 


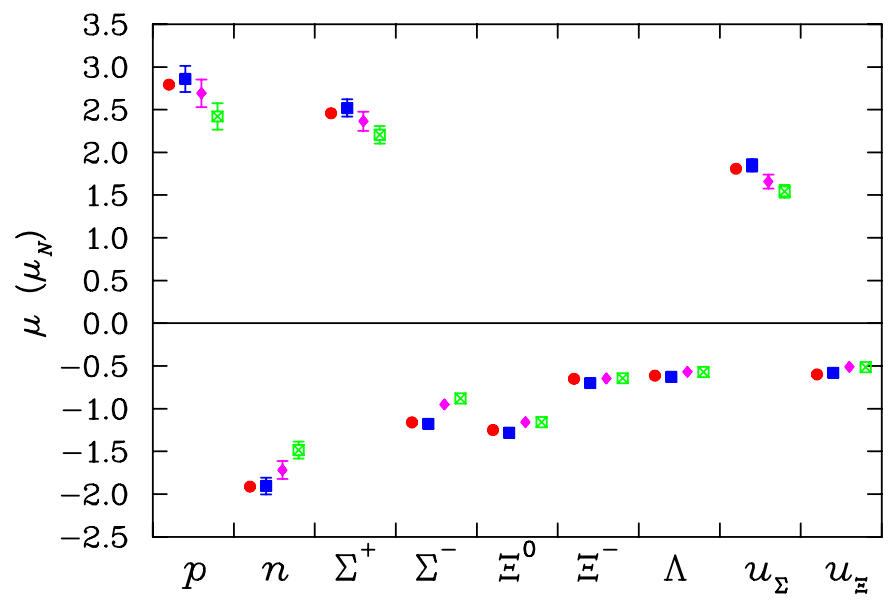

Fig. 3. The extracted full-QCD magnetic moments (ם) [16] display excellent agreement with the experimental moments $(\bullet)$. To indicate the size of corrections, the quenched $(\bullet)$ and finite-volume quenched $(\otimes)$ are also displayed.

Using the same procedure which enabled the correction from quenched to dynamical within the valence sector, we have also estimated the disconnected contributions to reconstruct the full magnetic moments in QCD. In Figure 3 the full magnetic moments are shown with the experimentally measured values for the entire baryon octet. The agreement with experiment is remarkable, offering further support for the validity of this analysis. Further, Figure 3 also displays our excellent reproduction of the two experimental valence moments, $u^{\Sigma}$ and $u^{\Xi}$.

With the valence ratios determined, the final input required from Eqs. (1) and (2) is the ratio ${ }^{l} R_{d}^{s}$. As discussed above, there have been technical difficulties in extracting a signal for the disconnected insertion directly. Therefore we have used the relative magnitude of the strange-tolight disconnected insertions, estimated through the same method that applied the unquenching corrections and constructed the full moments displayed in Figure 3. Given that the magnitude of the valence sector is so well reproduced, particularly by $u^{\Sigma}$ and $u^{\Xi}$, and that the overall scale of the disconnected insertions is observed to correctly adjust the full moments from the valence-only sector, we have substantial confidence that our estimate is accurate.

The final result for the strange magnetic moment of the proton is $G_{M}^{s}=-0.046 \pm 0.022 \mu_{N}$ [16], an error of just two hundredths of a nuclear magneton. The error quoted includes the best possible estimates of the statistical and systematic errors in the calculation [20]. The announcement by that G0 Collaboration that $G_{M}^{s}$ was positive, at the $95 \%$ confidence level [21], posed a significant challenge to this theoretical result.

Recently, the same techniques have been applied to theoretically extract the strange electric form factor of the proton [22]. The absence of accurate data for octet charge radii meant that it was necessary to use absolute values of the unquenched contributions of the $u$ and $d$ quarks to the charge radius of the proton. Again the result was particularly accurate, with $G_{E}^{s}\left(Q^{2}=0.1 \mathrm{GeV}^{2}\right)=+0.001 \pm$
$0.004 \pm 0.004$. This is in quite good agreement with the published measurement by the HAPPEx Collaboration at Jefferson Lab, $G_{E}^{s}\left(Q^{2}=0.1 \mathrm{GeV}^{2}\right)=-0.01 \pm 0.03$ [23].

With the increasing collection of strangeness measurements, and the promise of even more accurate data from HAPPEx in 2006, it is especially timely to see consolidated treatment of the strange form factor extraction. Recently, a systematic analysis of the published world data has been performed in Ref. [24]. The next section briefly summarizes the input to that analysis and its main conclusions.

\section{Global analysis of experiment}

Since the first results reported by the SAMPLE Collaboration in 1997 [25], many measurements of the parityviolating contribution to the elastic form factors of the nucleons have now been completed. These experiments have been performed on several targets, the proton, deuteron and helium-4, and at various kinematic configurations. The measured parity-violating asymmetries are sensitive to the strange electric and magnetic form factors in different linear combinations. By combining the results of these measurements, one can separate the electric and magnetic contributions.

In addition to probing the strange vector current, the experimental asymmetries are also sensitive to the the weak axial current in the nucleon, which become increasingly more significant at backward scattering angles. The axial- $Z$ coupling to the nucleon can be controlled through semileptonic decays, deep-inelastic scattering and careful treatment of radiative corrections. In addition, there is a parity-violating photon coupling, which is sensitive to an odd-parity component of the nucleon wavefunction the anapole form factor [26]. Being nonperturbative in origin, one is forced to introduce another unknown form factor which must be determined by data. Because a single stand-alone experiment is sensitive to several unknown form factors, the strangeness content has previously been reported by imposing theoretical estimates for the anapole contributions [27]. With the extensive experimental programs now completed, a global fit of all the data permits the extraction of all unknown form factors [24], without any need for theoretical input.

The parity-violating asymmetries have been constructed with a consistent set of inputs to avoid introducing any systematic distortion in the extracted form factors. In particular, the asymmetries have all be computed using the same set of electromagnetic form factors [28] and the latest radiative corrections [29].

The cleanest separation of the unknown form factors is available at $Q^{2} \sim 0.1 \mathrm{GeV}^{2}$, where the most substantial coverage of measurements have been performed [23,30]. With further data collected in the near $Q^{2}$-vicinity, particularly by the G0 Collaboration [21], it is useful to use as much of the available data as possible. Using measurements over a range of $Q^{2}$ values necessitates introducing a parameterisation of the $Q^{2}$ evolution of the form factors. A Taylor expansion of the strange electric and magnetic 
form factors has been utilised, defining

$$
G_{E}^{s}=\rho_{s} Q^{2}+\rho_{s}^{\prime} Q^{4}+\ldots, \quad G_{M}^{s}=\mu_{s}+\mu_{s}^{\prime} Q^{2}+\ldots .
$$

Provided the range of $Q^{2}$ values does not extend too high, this gives a systematic technique to combine a large set of data.

Because anapole contribution contributes together with the asymmetry arising from the axial charges, for simplicity the same dipole form is chosen for the axial and anapole contributions, with

$$
\tilde{G}_{A}^{N}=\tilde{g}_{A}^{N}\left(1+Q^{2} / M_{A}^{2}\right)^{-2},
$$

and

$\tilde{g}_{A}^{N}=\left(\xi_{A}^{T=1} g_{A} \tau_{3}+\xi_{A}^{T=0} a_{8}+\xi_{A}^{0} a_{s}\right)+\left(A_{\mathrm{ana}}^{T=1} \tau_{3}+A_{\mathrm{ana}}^{T=0}\right)$.

The factors, $\xi$, denote the tree-level plus radiative corrections multiplying the various axial charges. These charges are relatively well known, with $g_{A}=1.2695, a_{8}=0.58 \pm$ $0.03 \pm 0.12$ and $a_{s}=-0.07 \pm 0.04 \mp 0.05$. The second error in $a_{8}$ and $a_{s}$ denotes a correlated uncertainty allowing for potential violations of $\mathrm{SU}(3)$-flavour symmetry in semileptonic hyperon decay. The second bracketed term describes the anapole form factor, for which there is only limited phenomenological information. Zhu et al. [27] have estimated the magnitude and Maekawa et al. [31] the leading momentum dependence. The approach taken here, is to determine this contribution from the data, free from theory input. Given the size of the uncertainties of the charges, $\tilde{g}_{A}^{p}$ and $\tilde{g}_{A}^{n}$, the error is dominated by the poor knowledge of the anapole contributions.

The fit to the complete set of world PVES data at $Q^{2}<0.3 \mathrm{GeV}^{2}$ yields best-fit parameters

$$
\begin{aligned}
\tilde{g}_{A}^{p} & =0.05 \pm 1.38 \mp 0.29, \\
\tilde{g}_{A}^{n} & =2.61 \pm 2.27 \mp 0.37, \\
\rho_{s} & =-0.06 \pm 0.41 \mp 0.00 \mathrm{GeV}^{-2}, \\
\mu_{s} & =0.12 \pm 0.55 \pm 0.07,
\end{aligned}
$$

where the first error denotes the uncorrelated experimental uncertainty and the second the correlated uncertainty in the G0 experiment. The joint determination of the strangeness electric and magnetic form factors at $Q^{2}=0.1 \mathrm{GeV}^{2}$ is shown in Figure 4, where we also compare with the theoretical prediction described above.

The $68 \%$ and $95 \%$ confidence intervals for the determination of $G_{M}^{s}$ against $\tilde{G}_{A}^{p}$ is shown in Figure 5 and $\tilde{G}_{A}^{n}-\tilde{G}_{A}^{p}$ in Figure 6. This helps provide some picture as to the four-dimensional parameter space that is being constrained by the data. Within the experimental uncertainty on the determination of the axial form factors, Figure 4 shows the strangeness form factors mapping out a long, yet narrow, region of parameter space - including the point of vanishing strangeness. Going to the space of the axial form factors, within the strangeness determination, these are quite poorly constrained in comparison to the theoretical result of Zhu et al. [27]. Nevertheless, they are

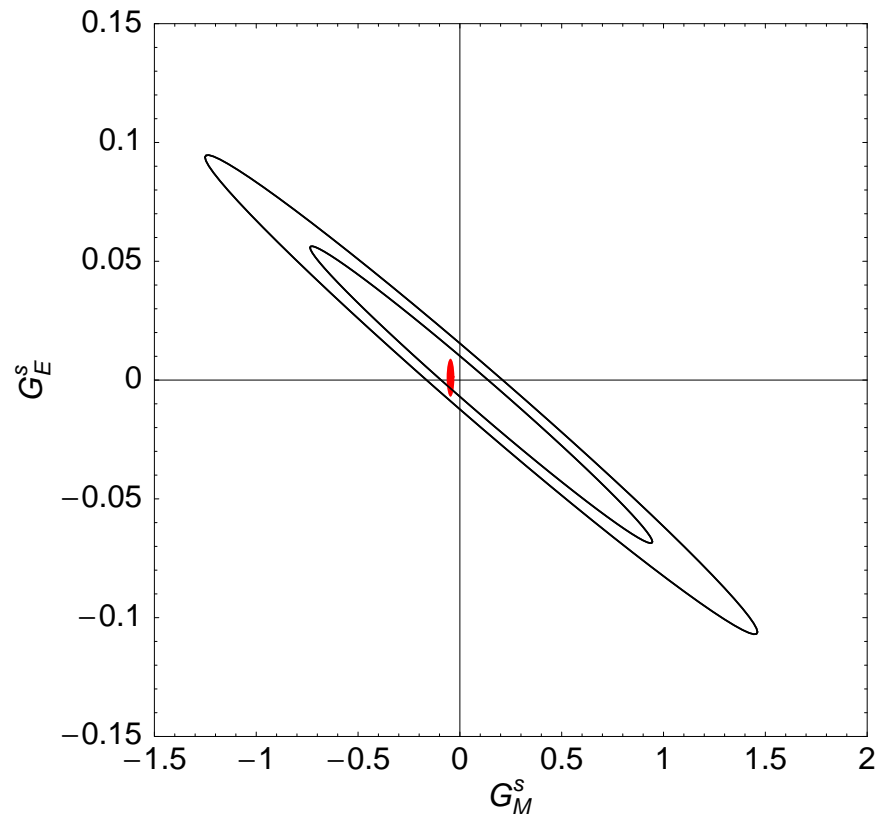

Fig. 4. Determination of the strangeness magnetic and electric form factors at $Q^{2}=0.1 \mathrm{GeV}^{2}, 68 \%$ and $95 \%$ confidence intervals are shown by the contours. The solid ellipse depicts the theory result described in Section 3 [16,22].

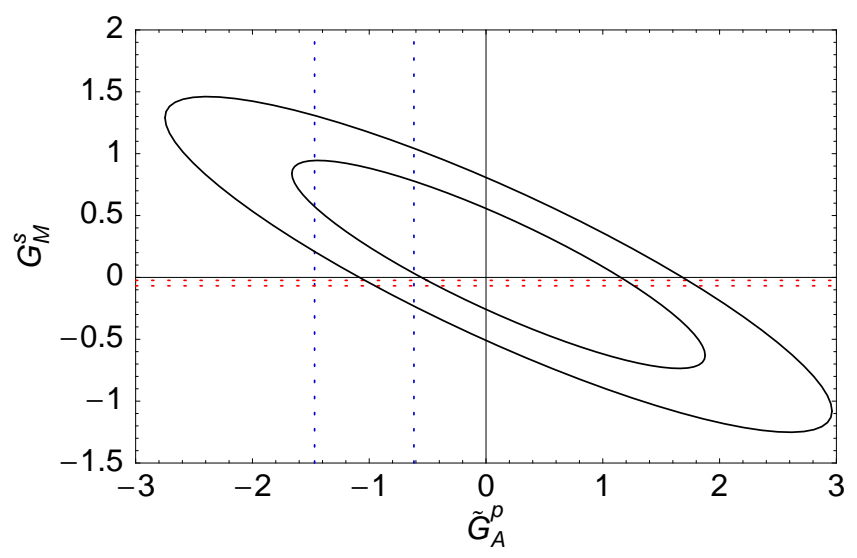

Fig. 5. The contours display the $68 \%$ and $95 \%$ confidence intervals for the joint determination of $\tilde{G}_{A}^{p}$ and $G_{M}^{s}$ at $Q^{2}=$ $0.1 \mathrm{GeV}^{2}$. The horizontal and vertical bands display the theory results of Leinweber et al. [16] and Zhu et al. [27], respectively.

totally consistent with this calculation, which includes the point of vanishing anapole form factor.

Independently of each other, the strangeness and anapole contributions appear to be consistent with zero, and hence in agreement with both the theoretical results in question. Figure 5 indicates that the two theory results appear to be outside the $68 \%$ confidence level to be simultaneously supported by the data. In the complete four-dimensional space, it is found that there is a $92 \%$ support for a nonzero value in at least one of the strange or anapole form factors [24]. 


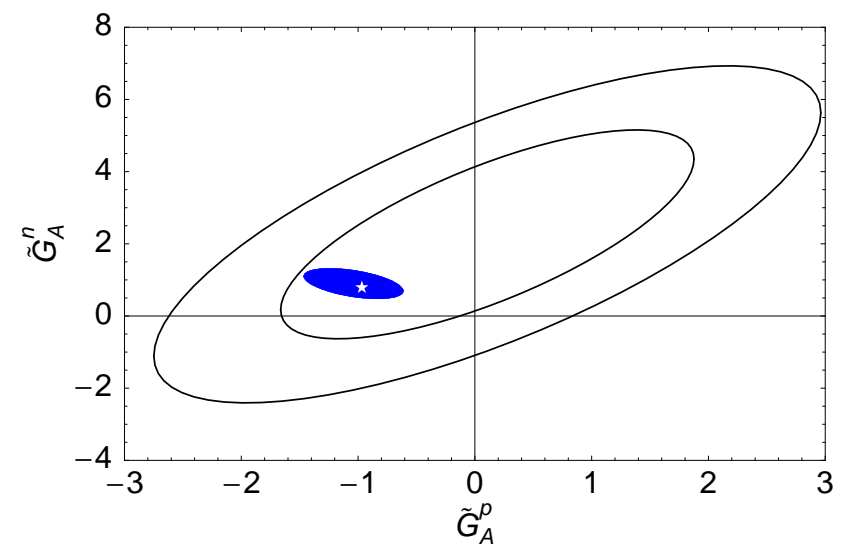

Fig. 6. The contours display the $68 \%$ and $95 \%$ confidence intervals for the joint determination of $\tilde{G}_{A}^{p}$ and $\tilde{G}_{A}^{n}$ at $Q^{2}=$ $0.1 \mathrm{GeV}^{2}$. The disc depicts the result of $\mathrm{Zhu}$ et al. [27], with the white star indicating a null anapole form factor.

\section{Summary}

A series of developments in the study of the chiral extrapolation problem in lattice $\mathrm{QCD}$, combined with a high-precision numerical calculation of baryon electromagnetic form factors, has enabled a detailed study of the strangeness content of the nucleon. A precise, small negative value has been predicted for the strangeness magnetic moment. The strangeness electric contribution is found to be bounded within half a percent of the total mean-square charge radius of the proton.

The determined strangeness form factors are found to be in good agreement with a global analysis of the world strangeness measurements. The anapole contributions in parity-violating elastic scattering are also consistent with modern theoretical estimates - although there is a small hint that both theory results cannot both be supported by the experimental data simultaneously. We look forward to future measurements which will further expand our view of the flavour structure of the nucleon.

\section{Acknowledgements}

Thanks are extended to the many collaborators who have been involved in the research presented here, particularly D. Leinweber and A. Thomas. This work was supported by U.S. DOE Contract No. DE-AC05-06OR23177, under which Jefferson Science Associates, LLC operate Jefferson Lab.

\section{References}

1. R. D. McKeown, Phys. Lett. B 219 (1989) 140; D. H. Beck, Phys. Rev. D 39 (1989) 3248.

2. R. G. Edwards et al. [LHPC Collaboration], Phys. Rev. Lett. 96 (2006) 052001 [arXiv:hep-lat/0510062].

3. C. Alexandrou et al., Phys. Rev. D 74 (2006) 034508 [arXiv:hep-lat/0605017].
4. M. Gockeler et al., arXiv:hep-lat/0609001.

5. R. D. Young et al., Prog. Part. Nucl. Phys. 50 (2003) 399 [arXiv:hep-lat/0212031].

6. S. R. Beane, Nucl. Phys. B 695 (2004) 192 [arXiv:heplat/0403030]. D. B. Leinweber et al., Nucl. Phys. A 755 (2005) 59 [arXiv:hep-lat/0501028]. V. Bernard and U. G. Meissner, Phys. Lett. B 639 (2006) 278 [arXiv:heplat/0605010]. J. A. McGovern and M. C. Birse, arXiv:heplat/0608002.

7. R. D. Young et al., Phys. Rev. D 71 (2005) 014001 [arXiv:hep-lat/0406001].

8. D. B. Leinweber et al., Phys. Rev. Lett. 92 (2004) 242002 [arXiv:hep-lat/0302020].

9. R. D. Young et al., Phys. Rev. D 66, 094507 (2002) [arXiv:hep-lat/0205017].

10. S. J. Dong et al., Phys. Rev. D 58 (1998) 074504 [arXiv:hep-ph/9712483]. N. Mathur and S. J. Dong, Nucl. Phys. Proc. Suppl. 94, 311 (2001) [arXiv:hep-lat/0011015]. R. Lewis et al., Phys. Rev. D 67 (2003) 013003 [arXiv:hep$\mathrm{ph} / 0210064]$.

11. W. Detmold, Phys. Rev. D 71 (2005) 054506 [arXiv:heplat/0410011]. F. X. Lee et al., Phys. Lett. B 627 (2005) 71 [arXiv:hep-lat/0509067].

12. J. Foley et al., Comput. Phys. Commun. 172, 145 (2005) [arXiv:hep-lat/0505023].

13. D. B. Leinweber, Phys. Rev. D 53 (1996) 5115 [arXiv:hep$\mathrm{ph} / 9512319]$.

14. G. A. Miller et al., Phys. Rept. 194 (1990) 1. B. Kubis and R. Lewis, Phys. Rev. C 74 (2006) 015204 [arXiv:nuclth/0605006].

15. D. B. Leinweber and A. W. Thomas, Phys. Rev. D 62 (2000) 074505 [arXiv:hep-lat/9912052].

16. D. B. Leinweber et al., Phys. Rev. Lett. 94 (2005) 212001 [arXiv:hep-lat/0406002].

17. X. Ji and D. Toublan, arXiv:hep-ph/0605055.

18. D. B. Leinweber, A. W. Thomas and R. D. Young, Phys. Rev. Lett. 86 (2001) 5011 [arXiv:hep-ph/0101211].

19. S. Boinepalli et al., arXiv:hep-lat/0604022.

20. D. B. Leinweber et al., Eur. Phys. J. A 24S2 (2005) 79 [arXiv:hep-lat/0502004].

21. D. S. Armstrong et al. [G0 Collaboration], Phys. Rev. Lett. 95 (2005) 092001 [arXiv:nucl-ex/0506021].

22. D. B. Leinweber et al., Phys. Rev. Lett. 97 (2006) 022001 [arXiv:hep-lat/0601025].

23. K. A. Aniol et al. [HAPPEX Collaboration], Phys. Lett. B 635, 275 (2006) [arXiv:nucl-ex/0506011]. K. A. Aniol et al. [HAPPEX Collaboration], Phys. Rev. Lett. 96 (2006) 022003 [arXiv:nucl-ex/0506010].

24. R. D. Young et al., to appear Phys. Rev. Lett. (2006) [arXiv:nucl-ex/0604010].

25. B. Mueller et al. [SAMPLE Collaboration], Phys. Rev. Lett. 78, 3824 (1997) [arXiv:nucl-ex/9702004].

26. W. C. Haxton et al., Phys. Rev. Lett. 63, 949 (1989).

27. S. L. Zhu et al., Phys. Rev. D 62 (2000) 033008 [arXiv:hep$\mathrm{ph} / 0002252]$.

28. J. J. Kelly, Phys. Rev. C 70, 068202 (2004).

29. S. Eidelman et al. [Particle Data Group], Phys. Lett. B 592, 1 (2004).

30. D. T. Spayde et al. [SAMPLE Collaboration], Phys. Lett. B 583 (2004) 79 [arXiv:nucl-ex/0312016]. F. E. Maas et al., Phys. Rev. Lett. 94 (2005) 152001 [arXiv:nucl-ex/0412030].

31. C. M. Maekawa et al., Phys. Lett. B 488 (2000) 167 [arXiv:hep-ph/0006181]. 